\title{
Optical and Electronic Properties of Monomers of Eumelanin: A DFT and TD-DFT Computational Study
}

\author{
R. Cardia ${ }^{1,2}$, G. Cappellini'1,2, E. Pinna, ${ }^{1,2}$, M. V. Tiddia ${ }^{1}$, G. Mula ${ }^{1,2}$ \\ ${ }^{1}$ Universitàdegli Studi di Cagliari, Dipartimento di Fisica, Cittadella Universitaria, Cagliari, Italy \\ ${ }^{2}$ Istituto Officinadei Materiali (CNR-IOM), UOS di Cagliari, Cittadella Universitaria, Cagliari, Italy \\ Email: giancarlo.cappellini@dsf.unica.it
}

Received 26 April 2016; accepted 20 August 2016; published 25 August 2016

\begin{abstract}
We report a systematic investigation on the electronic and optical properties of four monomers which are elementary constituents of some of the protomolecules of eumelanin. Eumelanin is the most important form of melanin which is one of the most universal natural pigments in living organisms. For the isolated monomers we performed all-electrons Density Functional Theory (DFT) and Time Dependent DFT (TDDFT) calculations with a localized Gaussian basis-set. For each monomer we determined a series of molecular properties, namely electron affinities, ionization energies, fundamental energy-gaps, optical absorption spectra, and exciton binding energies. We discuss moreover the possible implications of the above electronic and optical properties of the single monomers with respect to the properties of a recently proposed tetrameric protomolecule of eumelanin.
\end{abstract}

\section{Keywords}

Optical Properties, Biomolecules, TDDFT, Photochemistry, Photobiology, Optics in Biotechnology

\section{Introduction}

The molecule of eumelanin is the most diffuse biological kind of melanin and is the cause of several functions in living organism. In human body, eumelanin is responsible of the color of skin, hair and eyes. Aside from these facts, eumelanin results as an efficient photoprotective pigment. In fact, its broadband absorption [1]-[4] turns out to be efficient to defend living systems from intense sunlight [5] [6].

To provide an efficient photoprotective function, the spectrum of absorption of eumelanin has to be monotonically increasing toward the higher-energies. This broadband absorption spectrum is still an issue of scientific debate, particularly is not well understood how the molecular and aggregate structures of eumelanin relate to its overall optical features [7].

It has been demonstrated moreover that eumelanin also plays a role in some diseases as the Parkinson’s syn- 
drome [8], the macular degeneration [9] and the malignant melanoma [10].

All these facts make the study of the structural, electronic and optical properties of eumelanin of strategic important. Recent experiments have proved that eumelanin is made of stacked oligomers with an interlayer distance of $3-4 \AA$ [11]. X-ray diffraction studies [12] [13] and scanning tunneling microscopy measurements [14]-[16] demonstrated that the size of eumelanin protomolecules was of the order of 15 - $20 \AA$, which implied that the tetramers and pentamers formed by bonded DHIs monomers were the most probable molecular structures of eumelanin.

In this project we would like to address the computational characterization of different eumelanin monomers. Namely we shall consider IQ (indolequinone), MQ (quinone-methide), DHI (hydroquinone or 5,6-dihydroxyindole) and DHICA (5,6-dihydroxyindole 2-carboxylic acid) ones (see Figure 1). We shall use the all electrons Gaussian based code Nwchem which operates in the framework of Density Functional Theory (DFT) [17]. The electronic properties of the ground-state of each monomer will be addressed.

The ionization energy and electron affinity in the vertical and adiabatic form will be then calculated. Using then the Delta Self Consistent Field scheme $(\triangle \mathrm{SCF})$ we shall be able to calculate the quasiparticle gap of the systems under study. The use then of the time-dependent DFT (TD-DFT) which is implemented in the Nwchem package will enable us to calculate the absorption spectra. With the calculation of the above data we shall be also in the condition to evaluate the binding energy of the excitons for each system under study. Moreover starting from the more accepted models for the protomolecules of eumelanin [18] we shall try to identify which features in the electronic and optical properties of one of the possible eumelanin protomolecules could be assigned to its different constituent monomers.

Within the present study therefore we export a method successfully applied yet on several other families of organic molecules [19] [20] to another class of molecules which are the basic constituents of eumelanin.

\section{Computational Schemes}

We perform DFT and TDDFT computations using the NWChem package [17]. Geometry optimizations have been determined using the B3LYP hybrid exchange-correlation (XC) functional [21]-[23] and the PBE one [24], in combination with the $6-31+\mathrm{G}^{*}$ basis-set.

The use the B3LYP exchange-correlation (XC) functional has a rather standard behavior with some well documented limitations [25]. The structural optimization for each monomer has been performed without imposing symmetry constraints. The adiabatic electron affinities $\left(E A_{A}\right)$ and ionization energies $\left(I E_{A}\right)$ have been calculated via total energy differences. The vertical ionization energies $\left(I E_{V}\right)$ and electron affinities $\left(E A_{V}\right)$ have been calculated on the other hand at the relaxed geometry of the neutral molecules. This procedure permitted us the calculation of the quasi-particle energy gap which is rigorously defined in the $\triangle$ SCF scheme as [26] [27]:

$$
E_{\text {Gap }}=I E_{V}-E A_{V}=\left(E_{N+1}-E_{N}\right)-\left(E_{N}-E_{N-1}\right)
$$

To obtain then the singlet-singlet excitation energies and the electronic absorption spectrum in the visible/UV region for each monomer we performed TDDFT calculations at the same level B3LYP (PBE)/6 - $31+\mathrm{G}^{*}$ employed for its electronic ground-state. We used the frequency-space implementation of TDDFT founded on the linear response of the density-matrix. In this scheme the poles of the linear response function are associated to vertical excitation energies and the pole strengths to the corresponding oscillator strengths [28].

For each molecule we restricted ourselves to the first 50 singlet-singlet roots, which in all cases were sufficient to cover the energy window here considered $(3-7 \mathrm{eV})$. From the knowledge of the first optically active transition $E_{O p t}$ we could evaluate the exciton binding energy using the difference:
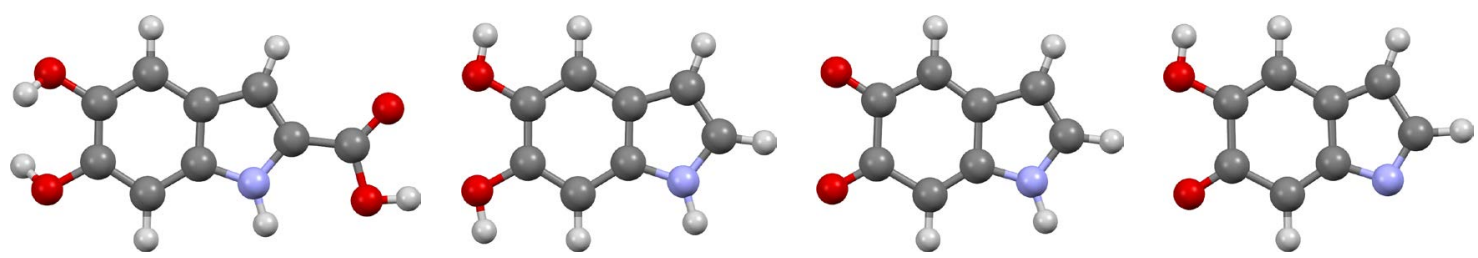

Figure 1. Monomers under study in the present paper, namely left DHICA, center-left DHI, center-right IQ and right MQ. In red oxygen atoms, in light blue nitrogen atoms, in white hydrogen atoms, in black carbon atoms. 


$$
E_{\text {Bind }}=E_{\text {Gap }}-E_{\text {Opt }} \text {. }
$$

Moreover, taking into account that orbital energy differences are in some cases fair approximations to electronic excitation energies, we compare the optical gap $E_{O p t}$ with the highest-occupied molecular orbital (HOMO) lowest unoccupied molecular orbital (LUMO) energy gap determined directly as difference of Kohn-Sham eigenvalues.

\section{Results and Discussion: Electronic and Optical Properties}

In Table 1 the energies which have been calculated in the present work, namely adiabatic and vertical ionization energies $\left(I E_{A}, I E_{V}\right)$, adiabatic and vertical electron affinities $\left(E A_{A}, E A_{V}\right)$, fundamental energy-gaps $\left(E_{G a p}\right)$, KohnSham gaps $\left(E_{K S g a p}\right)$, first optically active transition $\left(E_{\text {Opt }}\right)$ and exciton binding energy $\left(E_{\text {Bind }}\right)$, for the monomers under study. We used two different choices for the exchange and correlation potential, namely the B3LYP [21][23] and the PBE [24] ones. We consider first the case of the use of the XC potential B3LYP. The ionization energies $\left(I E_{A}, I E_{V}\right)$ have the largest values for the MQ monomer as the electron affinities $\left(E A_{A}, E A_{V}\right)$. On the opposite side we find the DHI monomer which shows the lowest values for the above quantities. For the fundamental gap and the optical onset energy are the DHI monomers which show the largest values. As a consequence for the exciton binding energy $E_{\text {Bind }}$ DHI reports the lowest value. The DHICA monomer is the other monomer which shows the values of the energies nearest to the ones of DHI. The tiny differences between DHI and DHICA data could be ascribed then to the presence of the $\mathrm{COOH}$ group. On the other hand the monomer IQ reports values for the observables similar to those of MQ. This behavior could be ascribed to the presence of the $\mathrm{OH}$ group which differentiates MQ with respect to IQ.

As is clear from Table 1 the use of PBE XC functional in some cases maintains the same ordering for the physical observables of the monomers (e.g. $I E_{A}, E A_{V}$ ) while for the other ones determines a change in the ordering with respect to the data after B3LYP XC calculations (e.g. $E_{\text {gap }}, I E_{V}$ ). These results demonstrate the importance and influence of the choice of the XC potential upon the electronicand optical properties of the monomers under study.

In Figure 2 and Figure 3 the spectra for the monomers here considered within TDDFT with the choices of PBE and B3LYP for the XC potentials.

In the case of DHI and DHICA the spectrum lies in the UV region with the use of both the XC potentials. On the other hand both IQ and MQ show absorption in the UV but are characterized also by smaller structures in the visible region either with the use of PBE and with the use of B3LYP XC potentials. To make a more quantitative comparison for the absorption spectra, in Table 2 we reported singlet-singlet permitted excitation energies (in

Table 1. Adiabatic and vertical ionization energies $\left(I E_{A}, I E_{V}\right)$, adiabatic and vertical electron affinities $\left(E A_{A}, E A_{V}\right)$, fundamental energy-gaps $\left(E_{\text {gap }}\right)$, Kohn-Sham gaps $\left(E_{K S g a p}\right)$, first optically active transition $\left(E_{O p t}\right)$ and exciton binding energy $\left(E_{\text {bind }}\right)$. All data are in $\mathrm{eV}$.

\begin{tabular}{ccccccccc}
\hline & \multicolumn{7}{c}{ B3LYP } \\
\cline { 2 - 8 } & $I E_{A}[\mathrm{eV}]$ & $I E_{V}[\mathrm{eV}]$ & $E A_{A}[\mathrm{eV}]$ & $E A_{V}[\mathrm{eV}]$ & $E_{\text {Gap }}[\mathrm{eV}]$ & $E_{K S g a p}[\mathrm{eV}]$ & $E_{\text {Opt }}[\mathrm{eV}]$ & $E_{\text {Bind }}[\mathrm{eV}]$ \\
\hline DHICA & 7.39 & 7.62 & 0.29 & -0.10 & 7.72 & 4.21 & 3.98 & 3.74 \\
DHI & 7.03 & 7.24 & -0.77 & -0.80 & 8.04 & 4.90 & 4.43 & 3.61 \\
IQ & 7.91 & 7.99 & 1.99 & 1.80 & 6.19 & 2.95 & 1.99 & 4.20 \\
MQ & 8.16 & 8.39 & 2.23 & 1.97 & 6.42 & 2.68 & 1.95 & 4.47 \\
& & & & & & & & \\
DHICA & 7.26 & 8.04 & 0.33 & -0.25 & 8.29 & 2.82 & 3.45 & 4.84 \\
DHI & 6.91 & 7.08 & -0.66 & -0.69 & 7.77 & 3.48 & 4.06 & 3.71 \\
IQ & 7.65 & 7.70 & 1.98 & 1.84 & 5.86 & 1.10 & 1.67 & 4.19 \\
MQ & 8.04 & 8.23 & 2.23 & 2.01 & 6.22 & 1.21 & 1.52 & 4.70 \\
\hline
\end{tabular}



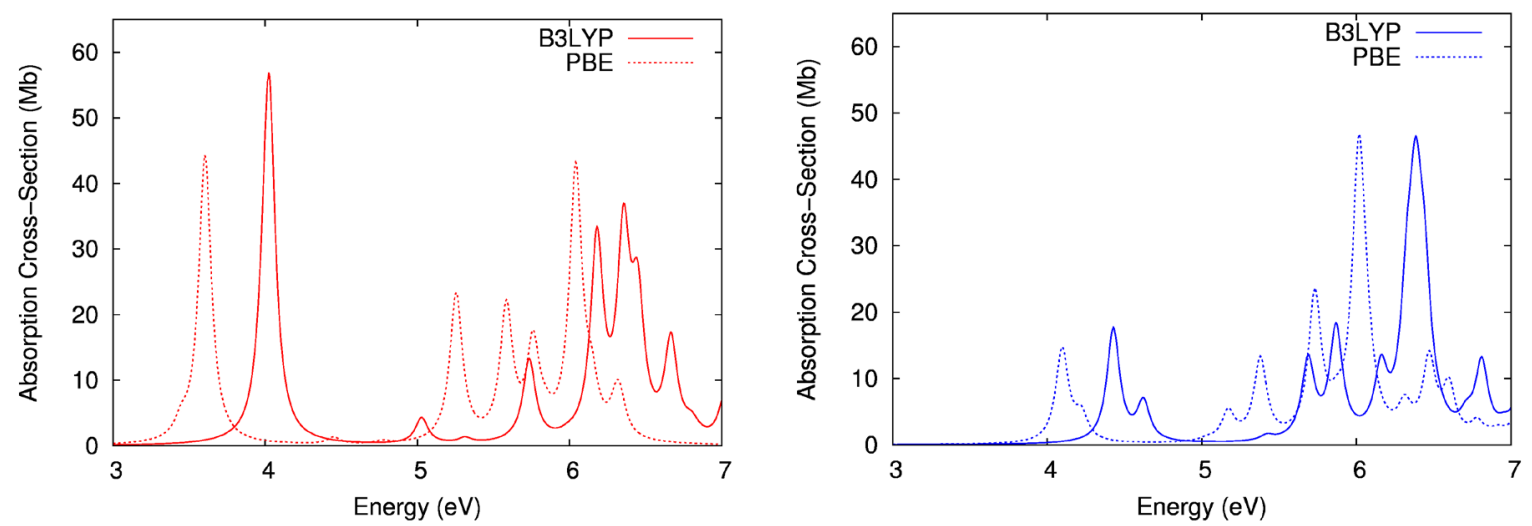

Figure 2. Absorption spectra for the DHICA (left) and DHI (right) monomers computed usingB3LYP (solid line) and PBE (dashed line) exchange-correlation functional.
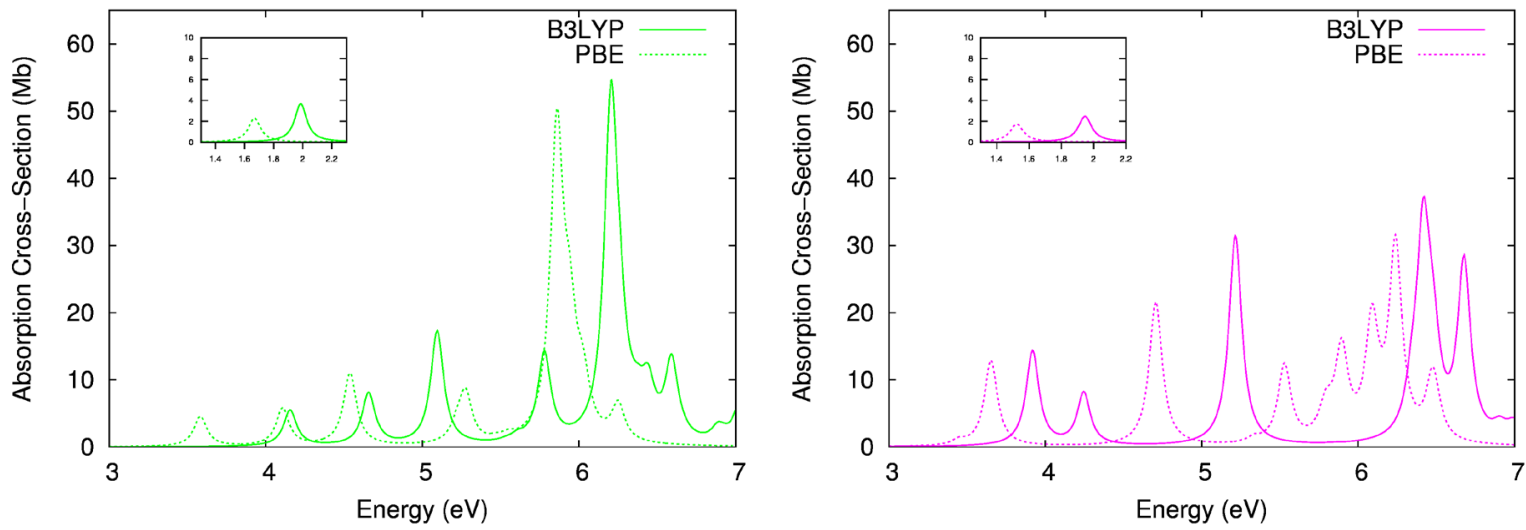

Figure 3. Absorption spectra for the IQ (left) and MQ (right) monomers computed usingB3LYP (solid line) and PBE (dashed line) exchange-correlation functional.

Table 2. Singlet-singlet permitted excitation energies (in eV) of eumelanin monomers as obtained by TDDFT using PBE/ B3LYP exchange correlation functional and $6-31+G^{*}$ basis set. The corresponding values of absorption cross-section (given between parentheses) are in Megabarn (Mb).

\begin{tabular}{cccc}
\hline & & B3LYP & \\
\cline { 2 - 4 } DHICA & Main Peak $[\mathrm{eV}]([\mathrm{Mb}])$ & First Optical-UV $[\mathrm{eV}]([\mathrm{Mb}])$ & Onset $[\mathrm{eV}]([\mathrm{Mb}])$ \\
DHI & $4.02(56.9)$ & $4.02(56.9)$ & $4.02(56.9)$ \\
IQ & $6.38(46.4)$ & $4.43(17.7)$ & $4.43(17.7)$ \\
MQ & $6.20(54.6)$ & $4.16(5.50)$ & $1.99(3.70)$ \\
& $6.42(37.3)$ & $3.92(14.4)$ & Onset $[\mathrm{eV}]([\mathrm{g})$ \\
& & PBE $])$ & $3.60(44.3)$ \\
DHICA & Main Peak [eV] $([\mathrm{Mb}])$ & $3.60(44.3)$ & $4.10(14.7)$ \\
DHI & $3.60(44.3)$ & $4.10(14.7)$ & $1.67(2.3)$ \\
IQ & $6.02(46.6)$ & $3.58(4.57)$ & $1.52(1.70)$ \\
MQ & $5.86(50.4)$ & $3.65(12.9)$ & \\
\hline
\end{tabular}


$\mathrm{eV}$ ) of eumelanin monomers as obtained by TDDFT using PBE/B3LYP exchange correlation functional and 6 $31+\mathrm{G}^{*}$ basis set. The corresponding values of oscillator strengths $f$ are given between parentheses. The energy range considered is $3-7 \mathrm{eV}$. In all the spectra the B3LYP XC data for the first absorption peak are blue-shifted by about $0.1-0.5 \mathrm{eV}$ with respect to those after PBE XC ones. For the DHICA monomer the strongest peak takes place at the onset.

On the other hand for the DHI the peak at onset results not the strongest one. For this monomer the largest structure comes in the UV. For IQ and MQ monomers, while the onset takes place in the visible, on the other side the more robust structures are placed in the UV. These strong absorption features in the UV for all the considered monomers is consistent with the typical absorption characteristics of all protomolecules of eumelanin [18].

Note please that the use of B3LYP XC potential causes different numbers and forms of the absorption structures at high energies in comparison with the PBE spectra.

In some cases for the monomers it is possible to perform a comparison with existing results in the literature. For the case of DHI the lowest-energy peak (around $4.1 \mathrm{eV}$ in energy or $301 \mathrm{~nm}$ in wave length) and the three structures in the range (around 5.2-5.7 eV or $216-240 \mathrm{~nm}$ ) in the absorption spectrum calculated using the PBE $\mathrm{XC}$ potential (see Figure 2) are in fair agreement (in energy position, relative energy distance and relative intensity) with the first two structures at low energy calculated within a different scheme (namely ZINDO/S method) used by Chun-Teh and coworkers [29]. Fair agreement is also found between the two lowest energy peaks in the absorption spectrum of MQ monomer after present calculations within the PBE-XC-scheme (see Figure 3), namely the first structure (around $3.7 \mathrm{eV}$ or $334 \mathrm{~nm}$ ) and the second one (around $4.7 \mathrm{eV}$ or $263 \mathrm{~nm}$ ), with those calculated by Chun-Teh and coworkers [29]. In the case of the monomer IQ, the present PBE-XC absorption spectrum (see Figure 3) compares well with that calculated previously within a similar scheme in the whole considered range (around $1.5-5.0 \mathrm{eV}$ or $250-800 \mathrm{~nm}$ ) by Meng and Kaxiras [18].

To test the utility and potentiality of our systematic approach for the electronic and optical properties of the monomers of eumelanin we then considered a proposed protomolecule of tetrameric structure named IMIM, made by two IQ and two MQ monomers assembled together [18].

In particular we decided to focus our attention to the UV region in which the two above monomers show intense absorption, i.e. 3.5 - $7 \mathrm{eV}$. In Figure 4 we report the absorption spectra in that energy region for the IQ and MQ monomers (with energy broadening of $0.07 \mathrm{eV}$ ) in comparison with the spectrum of IMIM after the calculation of Meng and Kaxiras [18].

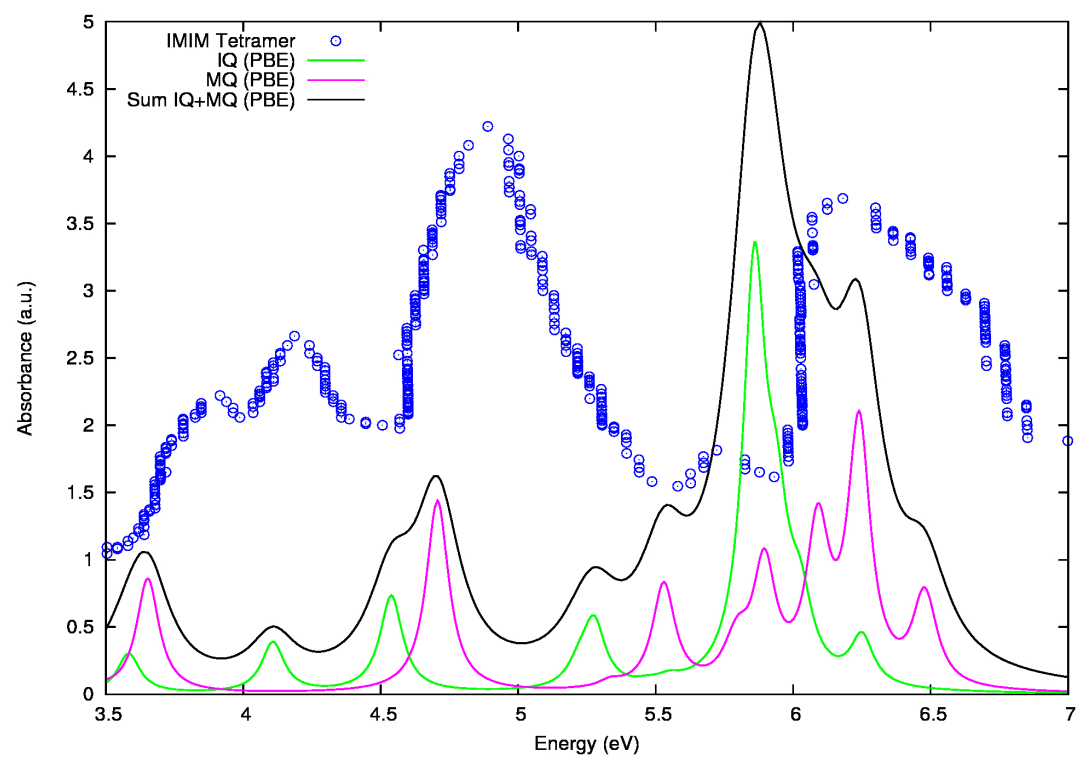

Figure 4. Absorption spectrum of IMIM tetramer proposed by Meng and Kaxiras in Ref. [18] (blue points), ideal IQ and MQ tetramers calculated spectra (green and blue respectively) and sum spectrum of IQ + MQ (black line), see text. For IQ, MQ and IQ + MQ the calculations are performed using PBE XC functional. 
We can indeed make a direct connection between the three main structure of IMIM and the peaks of the monomers IQ and MQ. Starting from the low energy part of the UV region depicted, the first structure of IMIM seems formed after the combination of the first peak of MQ and the first three peaks of IQ, the second structure of IMIM after the combination of the second and third peak of MQ and the fourth of IQ and finally the structure at high energy for IMIM after the summation of the structure 5 and 6 of IQ and 4 - 7 of MQ. In Figure 4 it is reported also the curve obtained as the sum of the spectra of MQ and IQ (with an energy broadening of $0.14 \mathrm{eV}$ ). This last curve reports the main three structures as in the results for the IMIM tetramer after Meng and Kaxiras [18] with a red-shift of the order of $0.3 \mathrm{eV}$.

This analysis suggests that in the important UV window $3.5-7 \mathrm{eV}$ the main absorption features of a recently proposed tetrameric protomolecule of eumelanin, namely the IMIM, could be ascribed to the absorption features of its single monomeric components.

\section{Acknowledgements}

The authors acknowledge the use of the computational facilities of the Center for Advanced Studies, Research and Development in Sardinia (CRS4) and of ISCRA-CINECA under project EUMELCOM.

This work was partially supported by the Regione Autonoma della Sardegna (RAS) base research project CRP78744 "Energy Applications with Porous Silicon (ENAPSi)”. G. C. and R. C. acknowledge useful discussions with G. Malloci. G. C. and R. C. acknowledge financial support from IDEA-AISBL-Bruxelles.

\section{References}

[1] Nofsinger, J.B., Forest, S.E. and Simon, J.D. (1999) Explanation for the Disparity among Absorption and Action Spectra of Eumelanin. Journal of Physical Chemistry B, 103, 11428-11432. http://dx.doi.org/10.1021/jp992640y

[2] Nofsinger, J.B. and Simon, J.D. (2001) Radiative Relaxation of Sepia Eumelanin Is Affected by Aggregation. Photochemistry and Photobiology, 74, 31-37. http://dx.doi.org/10.1562/0031-8655(2001)074<0031:RROSEI >2.0.CO;2

[3] Tran, M.L., Powell, B.J. and Meredith, P. (2006) Chemical and Structural Disorder in Eumelanins: A Possible Explanation for Broadband Absorbance. Biophysical Journal, 90, 743-752. http://dx.doi.org/10.1529/biophysj.105.069096

[4] Ou-Yang, H., Stamatas, G. and Kollias, N. (2004) Spectral Responses of Melanin to Ultraviolet A Irradiation. Journal of Investigative Dermatology, 122, 492-496. http://dx.doi.org/10.1046/j.0022-202X.2004.22247.x

[5] Brenner, M. and Hearing, V.J. (2008) The Protective Role of Melanin Against UV Damage in Human Skin. Photochemistry and Photobiology, 84, 539-549. http://dx.doi.org/10.1111/j.1751-1097.2007.00226.X

[6] Lister, T., Wright, P.A. and Chappell, P.H. (2012) Optical Properties of Human Skin. Journal of Biomedical Optics, 17, 0909011-09090115. http://dx.doi.org/10.1117/1.JBO.17.9.090901

[7] Meredith, P. and Sarna, T. (2006) The Physical and Chemical Properties of Eumelanin. Pigment Cell Research, 19, 572-594. http://dx.doi.org/10.1111/j.1600-0749.2006.00345.x

[8] Bush, W.D., et al. (2006) The Surface Oxidation Potential of Human Neuromelanin Reveals a Spherical Architecture with a Pheomelanin Core and a Eumelanin Surface. Proceedings of the National Academy of Sciences, 103, 1478514789. http://dx.doi.org/10.1073/pnas.0604010103

[9] Berendschot, T.T., Willemse-Assink, J.J., Bastiaanse, M., de Jong, P.T. and van Norren, D. (2002) Macular Pigment and Melanin in Age Related Maculopathy in a General Population. Investigative Ophthalmology Visual Science, 43, 1928.

[10] Pavel, S., et al. (2004) Disturbed Melanin Synthesis and Chronic Oxidative Stress in Dysplastic Naevi. European Journal of Cancer, 40, 1423-1430. http://dx.doi.org/10.1016/j.ejca.2003.11.035

[11] Chen, C.-T., et al. (2013) Self-Assembly of Tetramers of 5,6-Dihydroxyindole Explains the Primary Physical Properties of Eumelanin: Experiment, Simulation, and Design. ACS Nano, 7, 1524-1532. http://dx.doi.org/10.1021/nn305305d

[12] Cheng, J., Moss, S.C. and Eisner, M. (1994) X-Ray Characterization of Melanins I. Pigment Cell. Res., 7, $255-262$. http://dx.doi.org/10.1111/j.1600-0749.1994.tb00060.x

[13] Cheng, J., et al. (1994) X-Ray Characterization of Melanins II. Pigment Cell. Res., 7, 263-273. http://dx.doi.org/10.1111/j.1600-0749.1994.tb00061.x

[14] Zajac, G.W., et al. (1994) The Fundamental Unit of Synthetic Melanin: A Verification by Tunneling Microscopy of X-Ray Scattering Results. Biochimicaet BiophysicaActa (BBA)_General Subjects, 1199, 271-278. http://dx.doi.org/10.1016/0304-4165(94)90006-X 
[15] Zajac, G.W., Gallas, J.M. and Alvarado-Swaisgood, A.E. (1994) Tunneling Microscopy Verification of an X-Ray Scattering-Derived Molecular Model of Tyrosine-Based Melanin. Journal of Vacuum Science and Technology B, 12, 1512-1516. http://dx.doi.org/10.1116/1.587275

[16] Diaz, P., et al. (2005) Electrochemical Self-Assembly of Melanin Films on Gold. Langmuir, 21, 5924-5930. http://dx.doi.org/10.1021/la0469755

[17] Valiev, M., et al. (2010) NWChem: A Comprehensive and Scalable Open-Source Solution for Large Scale Molecular Simulations. Computer Physics Communications, 181, 1477-1489. http://dx.doi.org/10.1016/j.cpc.2010.04.018

[18] Meng, S. and Kaxiras, E. (2009) Theoretical Models of Eumelanin Protomolecule and Its Photoprotection Mechanism. Biophysical Journal, 94, 2095-2105. http://dx.doi.org/10.1529/biophysj.107.121087

[19] Cardia, R., et al. (2014) Effects of TIPS-Functionalization and Perhalogenation on the Electronic, Optical, and Transport Properties of Angular and Compact Dibenzochrysene. The Journal of Physical Chemistry A, 118, 5170-5177. http://dx.doi.org/10.1021/jp502022t

[20] Cardia, R., et al. (2016) Electronic and Optical Properties of Functionalized Polyaromatic Hydrocarbons: A Computational Investigation on Peruorinated Circumacenes. Proc. SPIE, 9895, 98950D. http://dx.doi.org/10.1117/12.2229744

[21] Becke, A.D. (1993) Density-Functional Thermochemistry. III. The Role of Exact Exchange. Journal of Chemical Physics, 98, 5648. http://dx.doi.org/10.1063/1.464913

[22] Lee, C.T., Yang, W.T. and Parr, R.G. (2 1988) Development of the Colle-Salvetti Correlation-Energy Formula into a Functional of the Electron Density. Phys. Rev. B, 37, 785-789.

[23] Stephens, P.J., et al. (1994) Ab Initio Calculation of Vibrational Absorption and Circular Dichroism Spectra Using Density Functional Force Fields. The Journal of Physical Chemistry, 98, 11623-11627. http://dx.doi.org/10.1021/j100096a001

[24] Perdew, J.P., Burke, K., and Ernzerhof, M. (1996) Generalized Gradient Approximation Made Simple. Phys. Rev. Lett, 77, 3865-3868.

[25] Grimme, S. and Parac, M. (2003) Substantial Errors from TDDFT for the Calculation of Excited States of Large Systems. Chem. Phys. Chem, 3, 292-295. http://dx.doi.org/10.1002/cphc.200390047

[26] Jones, R.O. and Gunnarsson, O. (1989) The Density Functional Formalism, Its Applications and Prospects. Rev. Mod. Phys, 61, 689-746.

[27] GMalloci, G., et al. (2004) Quasi-Particle Effects and Optical Absorption in Small Fullerene-Like GaP Clusters. Phys. Rev. B, 70, 205429.

[28] Casida, M.E. (1995) Time-Dependent Density Functional Response Theory for Molecules. In: Chong, D.P., Ed., Recent Advances in Density Functional Theory, Vol. I, World Scientic, Singapore. http://dx.doi.org/10.1142/9789812830586 0005

[29] Chen, C.-T., et al. (2014) Excitonic Effects from Geometric Order and Disorder Explain Broadband Optical Absorption in Eumelanin. Nature Communication, 5, Article Number: 3859. http://dx.doi.org/10.1038/ncomms4859

\section{Submit or recommend next manuscript to SCIRP and we will provide best service for you:}

Accepting pre-submission inquiries through Email, Facebook, LinkedIn, Twitter, etc.

A wide selection of journals (inclusive of 9 subjects, more than 200 journals)

Providing 24-hour high-quality service

User-friendly online submission system

Fair and swift peer-review system

Efficient typesetting and proofreading procedure

Display of the result of downloads and visits, as well as the number of cited articles

Maximum dissemination of your research work

Submit your manuscript at: http://papersubmission.scirp.org/ 